BMJ Paediatrics Open

\section{A Survey of implementation status of child nutrition surveillance systems, registry systems and information systems: a scoping literature review protocol}

Malihe Sadeghi, ${ }^{1}$ Mostafa Langarizadeh, ${ }^{2}$ Beheshteh Olang, ${ }^{3}$ Hamed Seddighi $(1),{ }^{4}$ Abbas Sheikhtaheri ${ }^{1}$

To cite: Sadeghi M, Langarizadeh $\mathrm{M}$, Olang $\mathrm{B}$, et al. A Survey of implementation status of child nutrition surveillance systems, registry systems and information systems: a scoping literature review protocol. BMJ Paediatrics Open 2021;5:e001164. doi:10.1136/ bmjpo-2021-001164

Received 20 May 2021 Accepted 3 August 2021
D) Check for updates

(c) Author(s) (or their employer(s)) 2021. Re-use permitted under CC BY-NC. No commercial re-use. See rights and permissions. Published by BMJ.

For numbered affiliations see end of article.

Correspondence to Hamed Seddighi; h.seddighi@ rug.nl

Dr Mostafa Langarizadeh; langarizade.m@iums.ac.ir

\section{ABSTRACT}

Introduction Child malnutrition in all forms is known globally as the leading cause of poor health. Planning and solving this challenge require sources that collect data accurately. Nutrition surveillance systems (NSS), nutrition registry systems (NRS) and nutrition information systems (NIS) collect and analyse data on nutrition status. Unfortunately, these systems only exist in a few countries. The methods that these systems use significantly differ and their effectiveness is also scarcely researched. This scoping literature review aimed to conduct a survey on NSS, NRS and NIS that collect data on children's nutrition at national and international levels, along with their attributes.

Methods and analysis The methods and analyses of this scoping review follow the Arksey and O'Malley's methodology. This scoping literature review will be conducted in five stages based on this method. (1) The main research question and subquestions are identified. (2) Relevant studies are extracted. In this step, we will search electronic databases including PubMed, Scopus and ISI Web of Science. A manual search will also be performed in Google Scholar, grey literature, and the websites of organisations such as WHO, UNICEF, Centers for Disease Control and Prevention, National Health Service, International Food Policy Research Institute, Food and Agriculture Organization, Food and Nutrition Technical Assistance, United Nations World Food Programme, and United Nations System Standing Committee on Nutrition. (3) Extracted studies are separately reviewed by two reviewers based on inclusion and exclusion criteria, and eligible studies are then selected. A third reviewer resolves disagreements. (4) A checklist is developed to extract the features. Data of included systems are separately extracted and entered into a checklist by two reviewers. A third reviewer then resolves any disagreement. (5) Data are summarised and analysed and are presented in tables and figures.

Discussion This scoping literature review provides strong evidence of the status of systems that collect data on the status of child nutrition. This evidence can help select best practices which can be applied to develop future systems. It can also be a positive step towards achieving an integrated system.

\section{What is already known on this topic?}

For global planning to improve child malnutrition and its problems, an integrated monitoring system is needed to collect and analyze data on nutrition status accurately.

As a prerequisite to achieving this system, it is recommended that the different types of nutrition monitoring systems which exist in some countries are identified.

\section{What this study hopes to add?}

This study prepares a list of nutrition surveillance systems, nutrition registry systems and nutrition information systems, including the purpose of system design, data collection methods, nutrition indicators used and minimum data set.

- The study provides information which can help select best practices that can be applied to develop future systems.

\section{INTRODUCTION}

In general, malnutrition in all forms is known as the leading cause of poor health. ${ }^{1}$ Many children suffer from malnutrition worldwide. ${ }^{2}$ Paediatric malnutrition is an imbalance between nutritional needs and intake, which leads to insufficient accumulation of energy, protein or micronutrients and negatively affects the child's growth and development. ${ }^{3}$

Malnutrition is a concept that encompasses undernutrition and overnutrition. ${ }^{4}$ Child undernutrition is generally referred to as a condition in which food intake is insufficient to meet the child's needs for growth, physiological function and dealing with a disease. ${ }^{5}$ On the other hand, overnutrition is the excessive intake of nutrients, which leads to fat accumulation in the body that would consequently threaten health. ${ }^{6}$ 
International studies show that undernutrition has always been the most common form of malnutrition in low-income and middle-income countries. As the population undergoes economic growth, urbanisation and demographic changes, overnutrition or overweight is emerging as a problem. Some areas experience both undernutrition and overnutrition, for example, Indonesia, Thailand, Southeastern China, Botswana, Cameroon and Central Nigeria. ${ }^{7}$

Malnutrition is also seen in children with stunting, wasting, overweight and obesity. Moreover, in 2019, 144 million children under 5 were stunted, 47 million children were wasted and 38 million were overweight. ${ }^{2}$ Research shows that malnutrition accounts for $45 \%$ of under-5 mortality worldwide, which is about 3.1 million deaths per year. ${ }^{8}$

On the other hand, the COVID-19 pandemic and its effects on food and nutrition, health and social protection systems are expected to significantly worsen malnutrition. ${ }^{9}$ Along with this, the pandemic is forecast to worsen undernutrition in low-income countries and obesity in middle-income and high-income countries. ${ }^{10}$

Globally, malnutrition has a destructive effect on various aspects of children's lives, including academic or professional growth, productivity at the society level and economic situation. Hence, it results in problems such as social exclusion and increased poverty. Malnutrition leads to a loss of $12 \%-15 \%$ of children's intellectual capacity. Malnourished children are also 8-12 times more likely than healthy children to suffer from chronic and infectious diseases because malnutrition induces an immunosuppressive effect that predisposes patients to recurrent infections. In children under 5 years of age, malnutrition leads to an increased risk of death, loss of language skills, physical dysfunction and weakened cardiovascular system. ${ }^{11}$

Malnutrition exerts intense financial pressure on individuals and health systems. ${ }^{12}$ The economic losses due to malnutrition have been reported in many studies. ${ }^{13} 14$ Economic models also indicate that the return on investment in nutrition has a robust cost to benefit ratio. ${ }^{15}$ Along with this, various investments in nutritional intervention programmes were conducted to reduce the incidence of child malnutrition. ${ }^{16-18}$

Accurate data on the nutrition status of children are required for timely nutritional interventions. Accurate data on the prevalence trends of malnutrition and early awareness of critical nutritional conditions can help in decision-making. For this purpose, continuous surveillance of children's nutrition status in different regions is necessary. ${ }^{19} 20$

The World Food Conference in Rome in 1974 recommended that various organisations such as WHO, Food and Agriculture Organization of United Nations and UNICEF establish systems for conducting surveys on the nutrition status of various population groups. ${ }^{21}$ Moreover, different systems were created in several countries to conduct surveys and surveillance on the nutrition status of different groups. ${ }^{22}$ These systems regularly collect nutrition data and provide information for examining trends and decisions. ${ }^{23}$

In addition, extensive surveys are usually conducted every few years and are useful tools to understanding national and global trends in malnutrition. ${ }^{23}$ The Demographic and Health Survey (DHS) is a comparable nationally representative household survey that has been conducted in some countries since 1984. The DHS collects a wide range of objective and self-reported data, focusing on fertility, reproductive health, child and maternal health, mortality, and nutrition. ${ }^{24}$

There are various reports of child malnutrition in different countries using DHS data. For example, in Ethiopia these surveys were used to show that children born to either overweight or educated mothers (elementary school, middle school or higher) have a lower risk of undernutrition. ${ }^{25}$ The incidence of stunting, wasting and underweight was $38.3 \%, 10.1 \%$ and $23.3 \%$, respectively. Approximately $19.47 \%$ of children were stunted and underweight and only $3.87 \%$ of children suffered from these three diseases at the same time in Ethiopia. ${ }^{26}$ In Pakistan, $44.4 \%$ of children under 5 years old were also stunted, $29.4 \%$ were underweight and $10.7 \%$ were wasted. ${ }^{27}$ Another example is Bolivia, which is currently at a transition stage, and is not only facing the problem of malnutrition but also facing the problem of overnutrition. $^{28}$

Some global surveys also depict geographical estimates of child malnutrition, providing a new horizon to policymakers, public health agencies and researchers to investigate and treat this universal childhood syndetic. ${ }^{7}$

Some studies investigated the effectiveness of nutrition surveillance systems (NSS) in a specific context. ${ }^{22} 232930$ In this regard, a report examined nutrition information systems (NIS) in several countries in crisis. ${ }^{31}$ To the best of our knowledge, no study has simultaneously investigated and compared these three systems, NSS, NIS and nutrition registry systems (NRS), with a focus on children at the national and international levels. Moreover, investigating the trends and planning on how to improve child malnutrition globally require integrated nutrition monitoring systems in countries that can uniformly gather information. To achieve this goal, first it is necessary to conduct a survey on the status of existing systems and identify their strengths and weaknesses. To do so, we therefore set out the present scoping literature review to conduct a survey on NSS, NRS and NIS, as well as DHS and surveys that use one of these systems to collect data on child nutrition.

\section{DEFINITION}

\section{Nutrition surveillance systems}

The NSS is a system coordinated with a central agency that regularly collects representative raw data on nutrition indicators and factors that influence decision-making. ${ }^{23}$ The NSS is used to identify nutrition and nutrition-related 
problems. These systems collect, analyse and report data on nutrition status, related diseases and risk factors in the population in a regular and timely manner. ${ }^{15}$

\section{Nutrition information systems}

The NIS is designed to promote health and nutrition status and reduce malnutrition. These systems collect, analyse and interpret nutrition and malnutrition information. Furthermore, by helping these systems, the quality of nutritional interventions is improved. ${ }^{32-34}$

\section{Nutrition registry systems}

A registry system for a disease is generally an organised system that uses observational research methods to collect consistent data (clinical and non-clinical) to assess specific outcomes for a population defined by a disease, condition or exposure, and serves one or more scientific, clinical or other predefined policies. ${ }^{35}$ An NRS is composed of observational data and nutritional interventions performed to continuously collect information. ${ }^{36}$

\section{PROTOCOL DESIGN}

The methodology used in this study is based on Arksey and O'Malley's scoping review methodology. ${ }^{37} 38$ According to this methodology, there are five stages involved in undertaking a scoping review: (1) identifying the research question(s); (2) identifying potentially relevant studies; (3) selecting eligible studies; (4) charting the data; and (5) collating, summarising and reporting the results.

\section{Stage 1: identifying the research question}

The key research question raised here is: what is the current implementation status of NSS, NRS and NIS for collection, evaluation and monitoring of data on children's nutrition status? The subquestions are as follows:

- What specific goals are considered for the design and development of these systems?

- What methodology is used to collect data in these systems?

- What criteria and indicators do these systems use to measure child malnutrition?

- What are the minimum data sets in these systems?

- What are the practical differences between these three systems (NSS, NRS and NIS) in the field of malnutrition?

\section{Stage 2: identifying relevant studies}

We will search electronic databases including PubMed, Scopus and ISI Web of Science for published literature. We will also conduct a manual search via Google Scholar and grey literature; the latter search will have no time limit and will continue until May 2021. To ensure that all relevant information is extracted, we will also search institutional websites of organisations such as the WHO, UNICEF, World Bank, Centers for Disease Control and Prevention, National Health Service, International Food Policy Research Institute, Food and Agriculture Organization, Food and Nutrition Technical Assistance,
Resource Library of Emergency Nutrition, World Public Health Nutrition Association, United Nations World Food Programme, and United Nations System Standing Committee on Nutrition.

The literature search strategy will include MeSH(Medical Subject Headings) terms and the following text keywords: (("status nutrition" OR malnutrition OR undernutrition OR emaciation OR undernourish* OR "protein-energy malnutrition" OR "protein calorie malnutrition" OR" protein deficiency*" OR subnutrition OR malnourished OR underweight OR overnutrition OR overweight) AND (registry OR register* OR "data management" OR "information management" OR surveillance OR "surveillance system" OR "monitoring system" OR "registry system" OR "minimum data set" OR "database" OR "Information system")).

An initial search will also be performed for the search strategy.

\section{Stage 3: study selection}

We will review the papers obtained from the search for duplicates and then remove duplicate papers. The review process will contain two stages of screening: (1) a title and abstract review and (2) a full-text review. In the first step, two reviewers will independently review the titles and abstracts of papers to assess eligibility based on the specified inclusion and exclusion criteria. The full-text assessment includes any paper considered important by each of the reviewers of the study. Two reviewers will independently analyse the full-text papers in the second phase to determine if they meet the inclusion/exclusion criteria. In the event of any dispute on the inclusion of a paper, the paper will be re-examined by the two reviewers, and if an agreement cannot be reached consultation with an impartial third reviewer will resolve the disagreement. The Preferred Reporting Items for Systematic reviews and Meta-Analyses extension for Scoping Reviews checklist will be used to develop this scoping review. ${ }^{39}$ Since this study attempts to analyse systems, the following inclusion and exclusion criteria will be applied to the systems described in the articles and reports:

\section{Inclusion criteria}

- Systems which collect data on malnutrition or nutrition status in the form of NSS, NRS and NIS.

- International and national systems in all countries.

- Ongoing systems or those with at least one completed data collection wave conducted periodically, that is, as a repetitive cross-sectional research.

- DHS collects data on children's nutrition in the form of one of these systems (NSS, NRS and NIS).

- Children as the main target population group (0-18 years old).

- All English and non-English full texts are considered.

Exclusion criteria

- Single cross-sectional, intervention and cohort studies. 
Table 1 Extracted data checklist

\begin{tabular}{|c|c|}
\hline Data heading & Description \\
\hline Name & System name \\
\hline Type & System type (NSS, NRS and NIS) \\
\hline Country(s) & $\begin{array}{l}\text { Name of the country or countries (in international systems) in which the system is } \\
\text { implemented }\end{array}$ \\
\hline Year & Year of system establishment \\
\hline Stakeholders & Organisations or institutions that support the system \\
\hline Key objectives & All the objectives and purposes for which the system was created \\
\hline Data collection centres & $\begin{array}{l}\text { Sources, centres and all places where data are collected, for example, from households, } \\
\text { schools, clinics }\end{array}$ \\
\hline Collected data & Minimum data set or information received from the patient in each system \\
\hline Malnutrition measurement criteria & $\begin{array}{l}\text { One way to measure malnutrition is to study nutrition-related outcomes, such as } \\
\text { anthropometric measurements, biochemical indicators, clinical signs of malnutrition, etc }\end{array}$ \\
\hline Population group & Age of children \\
\hline Geographical area covered & Cities or countries covered by each system \\
\hline Frequency of reporting & Number of times reports are given \\
\hline Frequency of data collection & Number of times and frequency of data being collected \\
\hline Website address & Website address of the system where it updates information about system activities \\
\hline
\end{tabular}

NIS, nutrition information systems; NRS, nutrition registry systems; NSS, nutrition surveillance systems.

- Adults or the elderly as the main target population group.

\section{Stage 4: data collection}

A checklist is developed to extract data and collect information on existing systems (NSS, NRS, NIS) (see table 1). The two reviewers will separately extract systems-related data and enter them into the checklist. Each reviewer's independently extracted data will be compared to ensure precise data collection. Any inconsistencies will also be further addressed to guarantee consistency among the reviewers. Thus, if there are any disagreements on the data, the attributes of the systems will be re-examined by the two reviewers, and if an agreement cannot be reached consultation with an impartial third reviewer will resolve the disagreement.

\section{Stage 5: data summary and synthesis of results}

The data are presented in tables and figures. A narrative report will be created to summarise the extracted data around the following results: list of different systems (NSS, NRS, NIS) that exist nationally and internationally, features and methodology of these systems, minimum data set of the systems, and differences among the systems examined according to their type. These results are explained according to the research question and the context of the overall purpose of study.

\section{Patient and public involvement}

Patients and/or the public were not involved in the design, or conduct, or reporting or dissemination plans of this research.

\section{DISCUSSION AND CONCLUSION}

This scoping literature review is performed to show the status of existing NSS, NRS and NIS that collect data on children's nutrition status. In some countries, these systems are designed and implemented to support the improvement of children's nutrition status. However, no attention has been paid to cataloguing the implementation status of these systems. This study provides a list of these systems, including the purpose of system design, the data collection methods, the nutrition indicators used and the minimum data set.

To achieve a world free from child malnutrition, we need an integrated nutrition monitoring system to classify and evaluate the geographical areas at risk of malnutrition. Through a uniform, accurate and timely information provided by this integrated system, authorities can provide comprehensive and effective policies to improve child malnutrition. Achieving an international agreement is essential in order to have an integrated system and facilitate data comparison in children. No doubt, examining nutrition data and the characteristics of existing systems can help select best practices which can be applied to develop future systems, which is a significant step towards reaching this agreement.

Furthermore, by examining the practical differences of these systems, their strengths and weaknesses can be identified, which can be helpfu for many countries that are looking for features of monitoring systems for children's nutrition status.

This study has some limitations. First, although some systems are active, they do not publish the results of their work, which makes it impossible for us to find them in 
our search. This can be taken as the main limitation of this protocol. The lack of precise information about the samples and transparency of the goals of the systems could be taken as a second limitation. Also, we think that non-uniformity in the quality and nature of these systems in terms of scope of work and the type of system is another limitation of the study.

Considering all the limitations, we hope that policymakers, healthcare providers and researchers use the results of this study in developing interventions to reduce child malnutrition, which is everyone's ultimate goal.

\section{Author affiliations}

${ }^{1}$ Department of Health Information Management, School of Health Management and Information Sciences, Iran University of Medical Sciences, Tehran, Iran ${ }^{2}$ Health Management and Economics Research Center, Health management research institute, Iran University of Medical Sciences, Tehran, Iran ${ }^{3}$ Pediatric Gastroenterology, Hepatology and Nutrition Research Center, Research Institute for Children's Health, Shahid Beheshti University of Medical Sciences, Tehran, Iran

${ }^{4}$ Campus Fryslân, University of Groningen, Leeuwarden, the Netherlands

\section{Twitter Hamed Seddighi @Seddighi.Hamed}

Contributors MS drafted the manuscript. ML, AS and BO critically reviewed the manuscript. HS contributed to the revision of the report. All authors contributed to the conception and design of the study and approval of the final version of the article.

Funding This study is part of MS's PhD thesis supported by the Iran University of Medical Sciences (grant no. 97-4-37-14267).

Competing interests None declared.

Patient consent for publication Not required.

Ethics approval This paper is part of MS's PhD thesis at the Iran University of Medical Sciences with ethics code IR.IUMS.REC.1397.1083 approved by the ethics committee of the university.

Provenance and peer review Not commissioned; externally peer reviewed.

Data availability statement № data are available. No data are available. All data relevant to the study will be included in the published journal article.

Open access This is an open access article distributed in accordance with the Creative Commons Attribution Non Commercial (CC BY-NC 4.0) license, which permits others to distribute, remix, adapt, build upon this work non-commercially, and license their derivative works on different terms, provided the original work is properly cited, appropriate credit is given, any changes made indicated, and the use is non-commercial. See: http://creativecommons.org/licenses/by-nc/4.0/.

\section{ORCID iD}

Hamed Seddighi http://orcid.org/0000-0002-6214-4830

\section{REFERENCES}

1 Swinburn BA, Kraak VI, Allender S, et al. The global syndemic of obesity, undernutrition, and climate change: the Lancet Commission report. Lancet 2019;393:791-846.

2 Level and trends in child malnutrition, UNICEF / WHO / World Bank Group Joint Child Malnutrition Estimates, Key findings of the 2020 edition. World Health Organization [internet], 2020. Available: https:// www.who.int/health-topics/malnutrition\#tab=tab_1 [Accessed 18 Feb 2021].

3 Dipasquale V, Cucinotta U, Romano C. Acute malnutrition in children: pathophysiology, clinical effects and treatment. Nutrients 2020;12:2413.

4 Khan A, Rasib Q, Ahmed H. Children mortality and malnutrition as a global issue. Clin Nutr 2019;38:1968-9.

5 Wells JCK, Briend A, Boyd EM, et al. Beyond wasted and stunted-a major shift to fight child undernutrition. Lancet Child Adolesc Health 2019;3:831-4.

6 KUASHIC B. Human malnutrition: twin burdens of undernutrition and overnutrition (nutrition and diet research progress. 1st edn. Nova Science Pub Inc, 2013.
7 LBD Double Burden of Malnutrition Collaborators. Mapping local patterns of childhood overweight and wasting in low- and middle-income countries between 2000 and 2017. Nat Med 2020;26:750-9.

8 Karunaratne R, Sturgeon JP, Patel R, et al. Predictors of inpatient mortality among children hospitalized for severe acute malnutrition: a systematic review and meta-analysis. Am J Clin Nutr 2020;112:1069-79.

9 Headey D, Heidkamp R, Osendarp S, et al. Impacts of COVID-19 on childhood malnutrition and nutrition-related mortality. Lancet 2020;396:519-21.

10 Zemrani B, Gehri M, Masserey E, et al. A hidden side of the COVID-19 pandemic in children: the double burden of undernutrition and overnutrition. Int J Equity Health 2021;20:1-4.

11 Mehta NM, Corkins MR, Lyman B. American Society for parenteral and enteral nutrition board of directors. defining pediatric malnutrition: a paradigm shift toward etiology-related definitions. $J$ Parenter Enteral Nutr 2013;37:460-81.

12 Okubo T, Janmohamed A, Topothai C, et al. Risk factors modifying the double burden of malnutrition of young children in Thailand. Matern Child Nutr 2020;16 Suppl 2:e12910.

13 Stein AJ, Qaim M. The human and economic cost of hidden hunger. Food Nutr Bull 2007;28:125-34.

14 Horton S, Steckel R. Malnutrition: global economic losses attributable to malnutrition 1900-2000 and projections to 2050 . How Much Have Global Problems Cost the Earth? A Scorecard from 1900 to. 2013 Oct 10, 2011: 2050. 247-72.

15 Horton S, Hoddinott J. Benefits and costs of the food and nutrition targets for the post-2015 development agenda post-2015 consensus. Copenhagen Consensus Centre, 2014.

16 Imdad A, Yakoob MY, Bhutta ZA. Impact of maternal education about complementary feeding and provision of complementary foods on child growth in developing countries. BMC Public Health 2011;11 Suppl 3:S25.

17 Penny ME, Creed-Kanashiro HM, Robert RC, et al. Effectiveness of an educational intervention delivered through the health services to improve nutrition in young children: a cluster-randomised controlled trial. Lancet 2005;365:1863-72.

18 Sawyer W, Ordinioha B, Abuwa P. Nutrition intervention program and childhood malnutrition: a comparative study of two rural riverine communities in bayelsa state, Nigeria. Ann Med Health Sci Res 2013;3:422-6.

19 Tuffrey V, Hall A. Methods of nutrition surveillance in low-income countries. Emerg Themes Epidemiol 2016;13:1-21.

20 UNICEF, Global Nutrition Cluster, Global Technical Assistance Mechanism for Nutrition. Nutrition information management, surveillance and monitoring in the context of COVID-19 (GTAM), 2020

21 Möhr M. Methodology of nutritional surveillance. Report of a joint FAO/UNICEF/WHO expert Committee. World Health Organ Tech Rep Ser 1976:1-6.

22 Quinn VJ, Kennedy E. Food security and nutrition monitoring systems in Africa: a review of country experiences and lessons learned. Food Policy 1994;19:234-54.

23 Tuffrey V. Nutritional surveillance systems, their use and value. London: Save the Children and Transform Nutrition, 2016.

24 Corsi DJ, Neuman M, Finlay JE, et al. Demographic and health surveys: a profile. Int J Epidemiol 2012;41:1602-13.

25 Kasaye HK, Bobo FT, Yilma MT, et al. Poor nutrition for under-five children from poor households in Ethiopia: evidence from 2016 demographic and health survey. PLoS One 2019;14:e0225996.

26 Tekile AK, Woya AA, Basha GW. Prevalence of malnutrition and associated factors among under-five children in Ethiopia: evidence from the 2016 Ethiopia demographic and health survey. BMC Res Notes 2019;12:1-6.

27 Khan S, Zaheer S, Safdar NF. Determinants of stunting, underweight and wasting among children $<5$ years of age: evidence from 20122013 Pakistan demographic and health survey. BMC Public Health 2019;19:358.

28 Miranda M, Bento A, Aguilar AM. Malnutrition in all its forms and socioeconomic status in Bolivia. Public Health Nutr 2020;23:s21-8.

29 Bel-Serrat S, Huybrechts I, Thumann BF, et al. Inventory of surveillance systems assessing dietary, physical activity and sedentary behaviours in Europe: a DEDIPAC study. Eur J Public Health 2017;27:747-55.

30 Friedman G. Review of national nutrition surveillance systems. Washington, DC: Food and Nutrition Technical Assistance III Project (FANTA), 2014.

31 Prendiville N. Nutrition and food security information systems in crisis-prone countries. FAO International Workshop on Food Security in Complex Emergencies, 2003: 23-5. 
32 Mock N, Mason J. Nutrition information systems for implementing child nutrition programs. Asian Dev Rev 1999;17:214-45.

33 Technology D. Overview of the nutrition information system in Uganda. Montpellier, France: Agropolis international, global support facility for the National information platforms for nutrition initiative. Report, 2019. Available: https://www.nipn-nutrition-platforms.org/ IMG/pdf/nutrition-info-system-uganda.pdf

34 Kuo S-E, Lai H-S, Hsu J-M, et al. A clinical nutritional information system with personalized nutrition assessment. Comput Methods Programs Biomed 2018;155:209-16.

35 Gliklich RE, Dreyer NA, Leavy MB. Registries for evaluating patient outcomes: A user's guide. Government Printing Office, 2014.
36 Braunschweig CA. Creating a clinical nutrition registry: prospects, problems, and preliminary results. J Am Diet Assoc 1999;99:467-70.

37 Daudt HML, van Mossel C, Scott SJ. Enhancing the scoping study methodology: a large, inter-professional team's experience with Arksey and O'Malley's framework. BMC Med Res Methodol 2013;13:48

38 Levac D, Colquhoun H, O'Brien KK. Scoping studies: advancing the methodology. Implement Sci 2010;5:69.

39 PRIZMA for Scoping Reviews. PRIZMA. Available: http://www. prisma statement.org/Extensions/ScopingReviews 